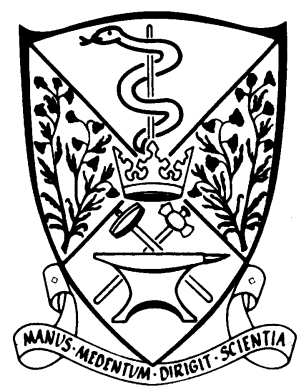

Department of Medicine, Hammersmith Hospital, London W12 0HS

Case presented by:

M K B Whyte, MRCP, medical registrar

\section{Chairman:}

C T Dollery, FRCP, professor of medicine

Discussion group:

A Adam, FRCR, consultant radiologist

P W Ind, MRCP, consultant physician (respiratory division)

Series edited by: Dr Robert Winter.

BrMed f 1989;299:1457-8

FIG 1 (right)-Posteroanterio chest radiograph showing $a$ subcarinal opacity with splaying of main bronchi

FIG 2 (below)-Computed tomogram showing 6 French dilator of Cope catheter introduction set lying extrapleurally, adjacent to cyst wall

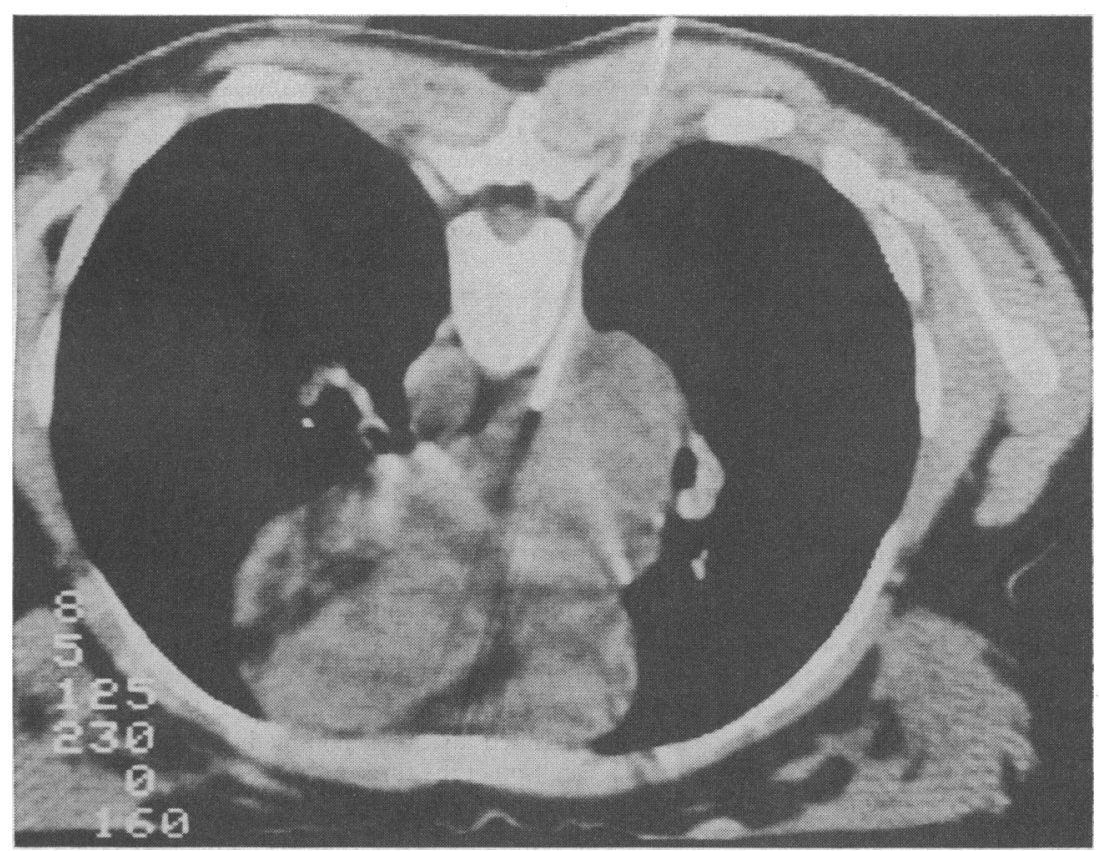

Bronchogenic cysts usually present as a chance finding in routine chest radiographs. Less commonly an increase in the size of or bleeding into the cyst may cause acute chest pain or respiratory embarrassment. We aspirated a large bronchogenic cyst under computed tomographic guidance by using a new extrapleural, percutaneous approach. This procedure was well tolerated and allowed complete drainage of the cyst.

\section{Case history}

A 24 year old medical physicist presented with a two week history of chest pain. She described the gradual

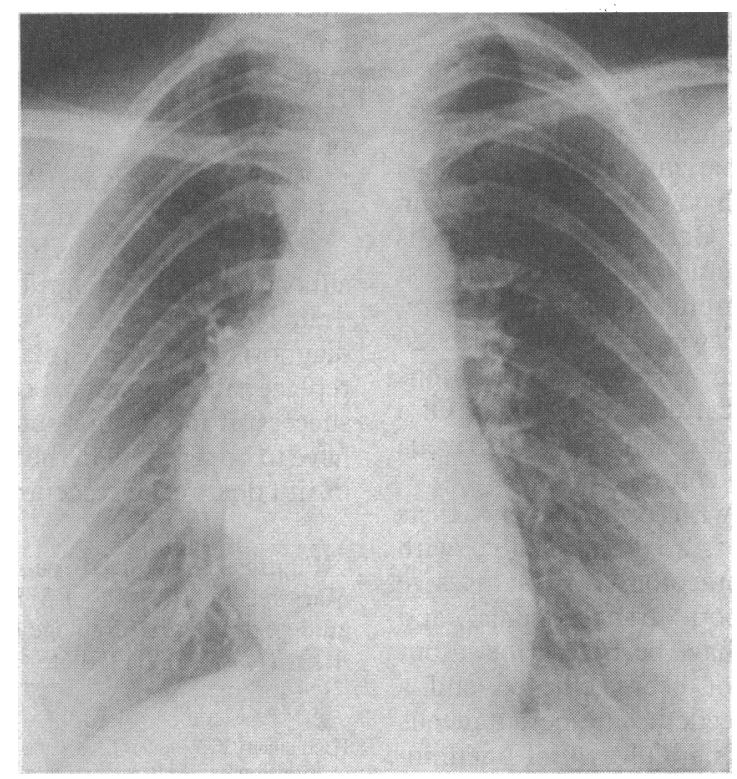

\title{
Central bronchogenic cyst: treatment by extrapleural percutaneous aspiration
}

\section{Satisfactory long term outcome after new non-operative procedure} enough to require regular oral analgesics, and a nonproductive cough. She had no history of cardiorespiratory disease and had never smoked. Physical examination yielded normal results.

Posteroanterior and lateral plain chest radiographs showed a large homogeneous opacity lying posteroinferior to the carina, displacing the heart anteriorly and splaying the carina (fig 1). A computed tomogram showed the opacity to be a unilocular cyst with a well defined wall, fulfilling the radiological criteria for a bronchogenic cyst. ${ }^{1}$ There was also a very small right pleural effusion. Her peak expiratory flow was reduced, being $3551 / \mathrm{min}$ or $78 \%$ of the predicted value, and maximum flow volume loops showed an abnormality of the expiratory limb, indicative of mild intrathoracic obstruction. Later, a copy was obtained of a routine chest radiograph taken one year before; careful inspection of this showed the cyst to be present but much smaller.

Aspiration of the central bronchogenic cyst was performed by extrapleural percutaneous aspiration. The patient lay on her front and a 21 gauge Chiba needle was advanced under computed tomographic guidance to just outside the parietal pleura. The needle was than advanced in a series of small steps, each preceded by injections of $2-5 \mathrm{ml}$ saline to separate the pleura from the chest wall. In this way the needle was guided down the space created until the cyst was reached. After introduction of a guide wire a number 6 French catheter was advanced into the cyst (fig 2), and $160 \mathrm{mls}$ of dark brown viscous fluid was drained. A computed tomogram immediately after aspiration (fig 3) showed almost complete disappearance of the cyst. The heart and great vessels, which had been displaced anteriorly by the cyst, resumed their normal position. The procedure was well tolerated by the patient, and there was no pneumothorax or other complication. Cytological examination of the cyst fluid showed numerous inflammatory cells with no evidence of malignancy, and all bacterial cultures including for acid fast bacilli yielded negative results. Six months after the procedure she was well and computed tomograms showed no evidence of reaccumulation of the cyst. Her peak expiratory flow rate and maximum flow volume loops were normal.

\section{Comment}

The oesophagus and the central bronchial tree share a common origin from the primitive endodermal yolk sac, and either may give rise to mediastinal cysts. Bronchogenic cysts arise by abnormal budding from the developing bronchial tree. As they are not usually detected at birth they are termed developmental rather than congenital. ${ }^{2}$ Central bronchogenic cysts account for $10 \%$ of all mediastinal masses ${ }^{3}$ and usually present in childhood or early adult life. There is no association with other congenital cystic diseases, and neoplastic change has not been described. Histologically, the cysts are lined with respiratory epithelium and are often thick walled, the walls containing fibrous tissue and sometimes cartilage. ${ }^{4}$ 


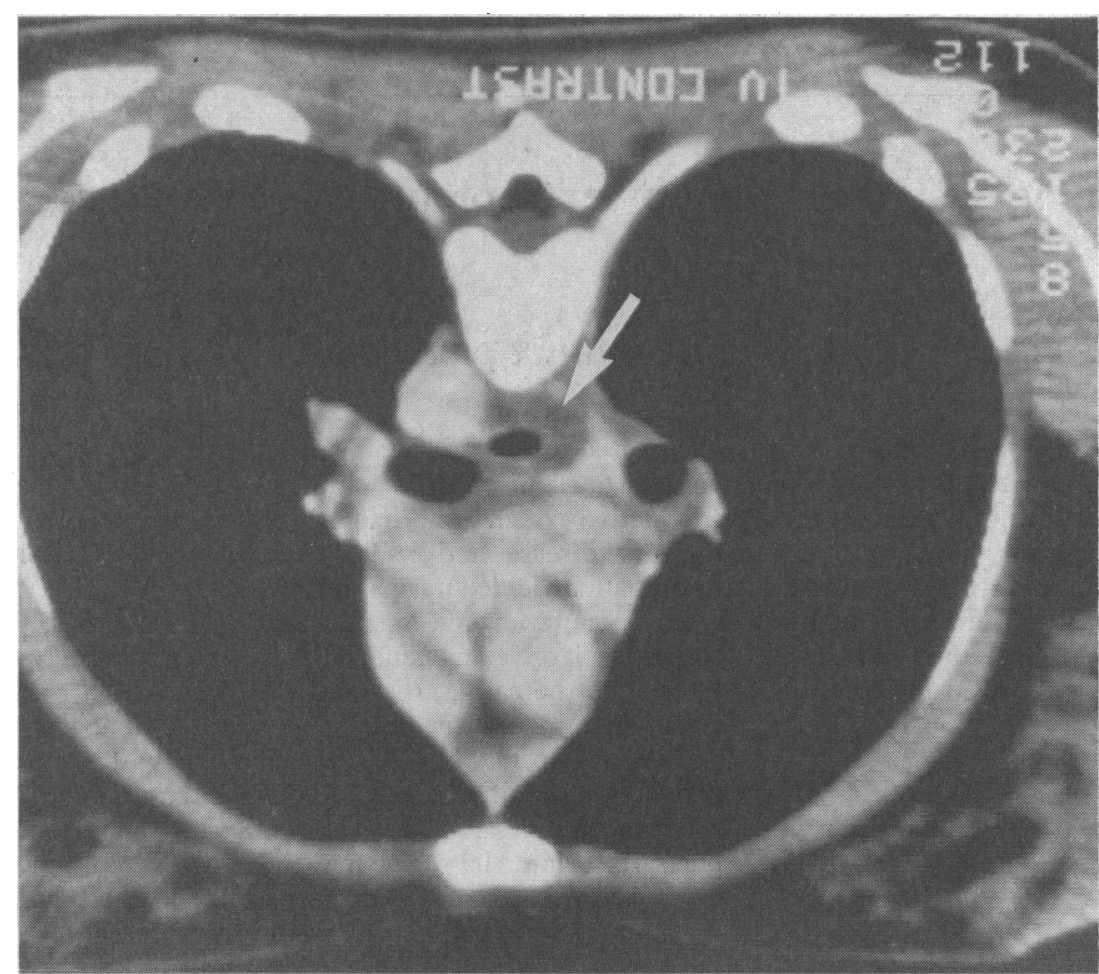

FIG 3-Computed tomogram after aspiration showing return of great vessels to their normal position and small amount of residual fluid around oesophagus (arrow). (Same orientation as fig 2 for comparison)

Most cases present as chance findings in routine chest radiographs. In this patient we suspect that a small leak from the cyst may have caused the chest pain that led to her presentation. Occasionally, symptoms can arise from infection or haemorrhage within the cyst or even rupture. These complications, though rare, may be life threatening: Kirwan et al described 26 patients of whom five had serious complications including acute stridor and cardiac tamponade. ${ }^{5}$ Of a series of 20 consecutive patients reported by Sirivella et al, two had life threatening complications. ${ }^{6}$

Until recently, patients with bronchogenic cysts have almost always been treated surgically, with removal of the cyst by thoracotomy. Less invasive means of treatment by both transbronchial and transoesophageal aspiration have been described, ${ }^{1}$ but complete evacuation has not been achieved and a general anaesthetic is still needed. The percutaneous transpulmonary route ${ }^{7}$ entails a high risk of pneumothorax and, again, complete aspiration has not been reported, probably because only fine needles can be used and the contents of the cyst are often viscous, as in this case. The methods we used allowed complete drainage because a catheter with a large internal diameter could be used and its tip manipulated to drain all the viscous contents of the cyst. Furthermore, this extrapleural approach requires only local anaesthetic and should be associated with a low risk of pneumothorax (because the pleura is not traversed). With this route the use of sclerosing agents to obliterate the cyst cavity could be considered in the event of recurrence.

\section{Discussion}

CTD: Why should the size of the cyst increase so much over one year?

PWI: The factors determining the growth of these developmental cysts are not well understood. In this patient the explanation that seems to fit best with her history is acute bleeding into the cyst: the fluid aspirated was dark brown in colour, and there was no evidence of infection. Her symptom of chest pain might have resulted from pressure on adjacent mediastinal structures or, to account for the small pleural effusion, a leak of fluid from the cyst.

CTD: Was this patient's management decided jointly with the cardiothoracic surgeons?

PWI: Yes, the plan of management was fully supported by the surgeons. All concerned felt that aspiration should be attempted as the patient would avoid a thoracotomy and its large, unsightly scar. The interesting point from the technical aspect of this case was the use of an extrapleural approach, avoiding the considerable morbidity of transoesophageal, transtracheal, and transpulmonary approaches. To our knowledge this technique had not been used previously and it is gratifying to see the patient well, without evidence of fluid recurrence, some time after the procedure.

Member of audience: May I ask what the patient was told about the procedure?

AA: The patient was told that she had a cyst that was almost certainly benign but that required removal or drainage to alleviate her symptoms and to confirm the diagnosis. It was explained that aspiration might replace a more major procedure but that it might not be successful and, even if successful on this occasion, may have to be repeated in the future as leaving the cyst wall in situ does make reaccumulation possible.

Figures reproduced with permission from Adam A Macsweeney JE, Whyte MKB, Smith PLC, Ind PW. CTguide extrapleural drainage of bronchogenic cyst. $\mathcal{F}$ Comput Assist Tomog 1989;13:1065-8.

I Kuhlman JE, Fishman EK, Wang KP, Zerhouni EA, Siegelman SS. Mediastinal cysts: diagnosis by CT and needle aspiration. American fournal of Radiolog 1988;150:75-8.

Anonymous. Bronchial cysts [Editorial]. Br Med f 1973;ii:501-2.

3 Morrison IM. Tumours and cysts of the mediastinum. Thorax 1958;13:294-6.

3 Morrison IM. Tumours and cysts of the mediastinum. Thorax

5 Kirwan WO, Walbaum PR, McCormack RJM. Cystic intrathoracic derivatives of the foregut and their complications. Thorax 1973;28:424-8.

6 Sirivella S, Ford WB, Zikna EA. Foregut cysts of the mediastinum: results in 20 consecutive surgically treated cases. $\mathcal{F}$ Thorac Cardiovasc Surg 1985;90:776 82 .

7 Fitch SJ, Tonkin ID, Tonkin AK. Imaging of foregut duplication cysts. Radiographics 1986;6:189-201

\section{NAMING NAMES}

\section{Dr Livingstone?}

Many patients regard their family doctor as a dear friend. Patients and doctors know Dr Livingstone to be the name of the missionary-explorer but how many are aware of the apt meaning of his name, "dear friend's place," from the Old English?

Let us explore for other surnames of medical interest.

The obvious start is with those that are descriptive of the profession. Leach, Leech, Leitch, and Leche are not difficult. All these arise from the Old English $l \bar{e} c e=$ doctor, Leitch being the Scottish form.

There is another group in Fisicien, Visick and Physick-as in "physician." Physick came to us through Old French from Latin.

The man acknowledged to be the father of American surgery was $\mathrm{Dr}$
Philip S Physick, born in Philadelphia in 1768. In recent years a chemist with the surname Physick was found trading in Adelaide, Australia.

Myers and Myer are derived from the Old French for a physician. The Mayers among us, in some instances, owe their name to the Old French maire, a town mayor, but where the origin is mire, meaning one who treats the sick, the name is firmly medical.

Surgeon, as an occupational surname, heads a group including Surgen, Surgenor, and Surgeoner. These often indicated a medical practitioner, but one whose craft was no means akin to modern surgery. Surgeon originated from the French surgien, itself from the Greek cheirourgia= handling, operating, and extended to other non-medical skills. The surname Mclay is said to mean "son of the surgeon" and is Scots Gaelic in origin. - BERNARD SHOUGH 